furosemide doses were associated with worse survival, identifying worse cases or suggesting a detrimental effect of over-diuresis. Lower blood pressure was also associated with decreased survival, probably because of lower cardiac output and worse cardiac disease. The relationship between heart failure, renal function and diuresis should be explored prospectively in larger study populations.

\section{The effect of heart disease on red cell mass}

\section{Madeleine Stein, Jenny Wilshaw, Nicola Lotter, Adrian Boswood}

Royal Veterinary College, London, United Kingdom

\section{OBJECTIVES}

This study aims to demonstrate whether a link exists between increasing severity of heart disease and red blood cell count in dogs with Myxomatous mitral valve disease.

\section{METHODS}

Data were obtained from 426 dogs enrolled in a longitudinal study from first opinion practices based in London between 2004 and 2017. PCV was compared between dogs at different American College of Veterinary Internal Medicine (ACVIM) stages of disease and factors related to changes in PCV were evaluated including; age, sex, breed, total protein, blood urea nitrogen, the left atrial to aortic root ratio and the normalised left ventricular internal diameter (LVIDDN).

\section{RESULTS}

One-way ANOVA showed a significant difference $(p<0.001)$ between all four ACVIM stages. Dogs in stage B2 $(41.12 \pm 6.15, p<0.001)$ had a significantly lower PCV than all other stages (A: $46.14 \pm 4.97, p<0.001$, B1: $43.44 \pm 5.80, p=0.012, C: 43.98 \pm 4.57, p=0.048)$. The results additionally demonstrated that the mean $P C V$ of dogs in stage A was the greatest and differed significantly from both stage B1 ( $p=0.007)$ and B2 $(p<0.001)$.

A multivariable linear regression analysis revealed that patient age $(-0.154 \pm 0.111, p=0.006)$, ACVIM stage $(-0.122 \pm 0.496, p=0.40), \operatorname{LVIDDN}(-0.160 \pm 1.122, p=$ $0.008)$ and breed $(0.471 \pm 0.661, p=0.001)$ all had $a$ significant and independent effect on PCV.

\section{STATEMENT (CONCLUSIONS)}

There is a link between mitral valve disease and red blood cell count in dogs, which differs from human disease. A decrease in red blood cell count is seen with diseased progression until patients enter congestive heart failure when there is an increase.

\section{A new hope? Transvascular pulmonic stents in six dogs with severe pulmonic stenosis}

\section{Kieran Borgeat ${ }^{1}$, Melanie Hezzell ${ }^{1}$, Guillaume Chanoit ${ }^{1}$, Jessie Rose Payne ${ }^{1}$, Pedro Oliveira ${ }^{2}$}

1 Langford Vets, University of Bristol, Bristol, United Kingdom

2 Davies Veterinary Specialists, Hitchin, United Kingdom

\section{OBJECTIVES}

Dogs with type B (hypoplastic) pulmonic stenosis have been traditionally considered poor candidates for minimally invasive treatment. We report six cases of type B pulmonic stenosis treated using pulmonic stents.

\section{METHODS}

Retrospective description of six dogs with pulmonic stenosis undergoing a transvascular pulmonic stent procedure in two cardiology referral centres, describing the approach and short-term outcomes.

\section{RESULTS}

Six dogs underwent a pulmonic stent procedure over a 12-month period. All dogs were diagnosed with severe, type B pulmonic stenosis based on echocardiographic criteria. Five French Bulldogs and one English Bulldog were treated. Two patients presented with signs of right sided congestive heart failure, two with erythrocytosis (concurrent right-to-left intracardiac shunts), one with syncope and one with marked exercise intolerance/ lethargy. Five dogs successfully received balloonexpandable metallic stents via a venous approach. In one dog, the stent could not be positioned appropriately and a hybrid procedure was successfully performed to place the stent retrograde, via the left pulmonary artery. No major complications were reported, although all dogs experienced reduced cardiac output and arrhythmias during stent deployment. All dogs experienced an improvement in clinical signs at one month post-operatively. 


\section{Oral presentations}

\section{STATEMENT (CONCLUSIONS)}

Pulmonic stents represent a new frontier in interventional cardiology to manage dogs with type B pulmonic stenosis that previously were considered poor candidates for minimally invasive procedures.

\section{Infiltrative laryngeal disease in 11 dogs}

\section{Amy Dixon ${ }^{1}$, Michael S. Tivers ${ }^{2}$, Leo Packham ${ }^{1}$, Vicki Black ${ }^{1}$}

1 Bristol Vet School, Bristol, United Kingdom

2 Paragon Veterinary Referrals, Wakefield, United Kingdom

\section{OBJECTIVES}

To describe the clinical features and outcome of infiltrative laryngeal disease in dogs.

\section{METHODS}

Medical records at a single referral centre (2013-2018) were retrospectively reviewed for dogs with infiltrative laryngeal disease diagnosed by cytology and/or histopathology. Referring veterinarians were contacted for follow-up.

\section{RESULTS}

Eleven dogs were included, with a median age of 5 years (range 1-14 years). Signs included inspiratory noise $(n=5)$, cough $(n=5)$ and dysphagia $(n=4)$, with a median duration of 6 weeks (1-16 weeks).

Laryngeal lesions were unilateral in eight dogs and bilateral in three. Ten dogs were diagnosed with inflammatory disease including neutrophilic $(n=3)$, septic neutrophilic $(n=1)$, granulomatous $(n=2)$, eosinophilic ( $n=1)$, lymphocytic/plasmacytic $(n=1)$ and mixed $(n=2)$. Three dogs underwent surgical excision of the lesion with adjunctive prednisolone $(n=2)$ or firocoxib $(n=1)$. Four dogs received corticosteroids without surgery (+/- antimicrobials) and one received antimicrobials alone. One dog was diagnosed with large cell lymphoma and was treated with chemotherapy. Nine dogs survived to discharge.

Follow-up was available for eight dogs diagnosed with inflammatory disease. Three had fully recovered (1, 23 and 32 months), one dog was receiving prednisolone ( 2 months) and four dogs had recurrence of clinical signs at 1, 5, 17 and 26 months, of which two were euthanased. The dog with lymphoma was in remission at 8 months.

\section{STATEMENT (CONCLUSIONS)}

Dogs can suffer infiltrative disease of the larynx and, in this cohort, inflammatory disease was more common than neoplasia. For dogs that survived to discharge outcome was fair although relapse was possible. 